\title{
Augmenting the space domain awareness ground architecture via decision analysis and multi-objective optimization
}

\author{
Albert Vasso and Richard Cobb \\ Department of Aeronautics and Astronautics, Air Force Institute of Technology, \\ Wright-Patterson AFB, Ohio, USA \\ John Colombi \\ Department of Systems Engineering, Air Force Institute of Technology, \\ Wright-Patterson AFB, Ohio, USA, and \\ Bryan Little and David Meyer \\ Department of Aeronautics and Astronautics, Air Force Institute of Technology, \\ Wright-Patterson AFB, Ohio, USA
}

\begin{abstract}
Purpose - The US Government is challenged to maintain pace as the world's de facto provider of space object cataloging data. Augmenting capabilities with nontraditional sensors present an expeditious and lowcost improvement. However, the large tradespace and unexplored system of systems performance requirements pose a challenge to successful capitalization. This paper aims to better define and assess the utility of augmentation via a multi-disiplinary study.

Design/methodology/approach - Hypothetical telescope architectures are modeled and simulated on two separate days, then evaluated against performance measures and constraints using multi-objective optimization in a heuristic algorithm. Decision analysis and Pareto optimality identifies a set of high-performing architectures while preserving decision-maker design flexibility.

Findings - Capacity, coverage and maximum time unobserved are recommended as key performance measures. A total of 187 out of $10^{17}$ architectures were identified as top performers. A total of $29 \%$ of the sensors considered are found in over $80 \%$ of the top architectures. Additional considerations further reduce the tradespace to 19 best choices which collect an average of 49-51 observations per space object with a 595-630 min average maximum time unobserved, providing redundant coverage of the Geosynchronous Orbit belt. This represents a three-fold increase in capacity and coverage and a $2 \mathrm{~h}$ $(16 \%)$ decrease in the maximum time unobserved compared to the baseline government-only architecture as-modeled.
\end{abstract}

(C) In accordance with section 105 of the US Copyright Act, this work has been produced by a US government employee and shall be considered a public domain work, as copyright protection is not available. Published in Journal of Defense Analytics and Logistics. Published by Emerald Publishing Limited.

Gratitude is extended to 14 WS, AFIT's Center for Directed Energy (CDE), AGI and the SDA community for help in various aspects of the M\&S. Developers of the software routines used in this study are also thanked for their dedication to providing high-quality, reliable tools whose usage underpinned and sped the completion of this study.

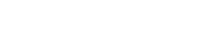


$\underset{5,1}{\mathrm{JDAL}}$

78

Originality/value - This study validates the utility of an augmented network concept using a physicsbased model and modern analytical techniques. It objectively responds to policy mandating cataloging improvements without relying solely on expert-derived point solutions.

Keywords Modeling and simulation, Multi-objective optimization, Space domain awareness, System architecting, Value-based decision-making

Paper type Research paper

\section{Introduction}

Through a worldwide network of ground-based telescopes and radars as well as spacebased optical platforms, the US Government (USG) collects and processes data on over 23,000 satellites, rocket bodies and pieces of debris with a diameter of $10 \mathrm{~cm}$ or greater in orbit (Lal et al., 2018). These Resident Space Objects (RSOs) are present in both the Near Earth and Deep Space orbital regimes. Deep Space RSOs are categorized as those with periods greater than $225 \mathrm{~min}$ and include RSOs in Geosynchronous Orbit (GEO) (USSTRATCOM, 2004a).

The general knowledge gleaned from collection of objects is referred to as Space Domain Awareness (SDA), which consists of both cataloging and characterization of RSOs. Cataloging includes identifying particular RSOs and projecting their future locations using astrodynamics equations; characterization ascribes properties, behavior and intent to the RSO. A second, related mission is Space Traffic Management (STM), which involves communicating conjunction probabilities to RSO owners and operators to prevent collisions and adding additional debris to congested operating areas.

For several historical reasons, the USG has become the world's de facto provider of no-cost, basic RSO cataloging and STM information. Multiple users rely on this data to ensure their defense, civil and commercial missions remain executable. Any disruption or inability to provide timely, rigorous information increases the risk of loss of mission. Disruptions in any space-based service - communications, timing, navigation, imagery collection - will impact users worldwide and imposes ripple effects to multiple users. Additionally, repeated failures to modernize equipment coupled with the projected increase in future space services and a shifting focus on new threats seriously jeopardizes the USG's ability to maintain space superiority and ensure safe STM for the world. National-level policy outlined in Space Policy Directive 3 (SPD-3) now mandates improving this situation.

Fortunately, nontraditional sensor providers have matured to the point where their contributions are routine, reliable and prime for incorporation into USG SDA processes. These providers are defined as one-off civil and scientific sensors and well-developed worldwide commercial tracking networks. The USG has recognized the utility of these capabilities as well as outlined how to vet these providers for future incorporation. However, no framework exists to assess the future system of systems of USG/nontraditional sensors, herein referred to as the Augmented Network (AN), nor how to approach designing the AN in an objective, optimal manner.

This study assumes a notional future scenario in which a USG Systems Program Office (SPO) is charged with designing the AN. The SPO will incorporate Deep Space cataloging data from one civil, one scientific and a set of sensors from three distinct commercial companies proposing a total of 56 sensors. Civil and scientific data from two large telescopes is assumed to be contributed for free without any ability to influence taskings. The SPO is assumed to be allocated $\$ 25 \mathrm{~m}$ to acquire some portion of commercial capabilities and decides to purchase exclusive rights to fully task and incorporate cataloging data from small-aperture, Narrow Field of View (NFOV) telescopes. 
The challenge becomes determining how to objectively select which sensors to include. Comparisons to source documents and other studies prompt three key performance measures be pursued: capacity, amount of time unobserved and coverage. Various AN permutations, or architectures, are modeled and simulated on two different nights and assessed using Multi-Objective Optimization (MOO) to evaluate performance subject to the cost constraint. A heuristic algorithm is used to smartly assess the tradespace of $10^{17}$ possible architectures. Pareto domination is applied post priori to identify the top performing architectures, while additional management-based considerations further reduces the tradespace. The decision-maker is left with a handful of choices culled from the best-performing architectures and higher confidence that a high-performing choice has been selected than by merely relying on a Subject Matter Expert's proposed point design.

\section{Background}

System architecting, decision analysis and optimization

Maier and Rechtin define the discipline of systems architecting as the art and science of creating and building complex systems through use of qualitative heuristic principles and quantitative analytical techniques (Maier and Rechtin, 2010). System needs and requirements permit the architect to define potential architectures, while measures help capture performance (Crawley et al., 2016). The evaluation of alternatives is aided by decision analysis. A common decision analysis approach uses values-focused thinking, which uses decision-maker objectives to quantify the performance of alternatives (Parnell et al., 2013).

Many problems have competing goals, complicating comparisons. Typical comparisons use either a weighted sum or MOO to illuminate the best performers. Crawley et al. recommend using MOO to evaluate a system's design tradespace, which is defined as "numerous architectures, represented at lower fidelity and evaluated with a few simple key metrics," and using Pareto and near-Pareto optimality to identify solutions (Crawley et al., 2016). Pareto optimality consists of ranking competing solutions based on their ability to outperform others in all objectives into successive fronts, where solutions on the same front do not outperform each other in all objectives. Rhodes and Ross outline an additional use of Pareto optimization in architecting via Epoch-Era Analysis, in which a system is assessed during multiple periods of fixed needs and contexts throughout its lifespan such that highperforming architectural choices common to all periods are posited as those with greater value (Rhodes and Ross, 2010).

\section{Astrodynamics, scheduling and optical collection}

Because of limitations in sensor capabilities, approximations in equations and models and measurement errors, an RSO's true position and velocity is rarely known. The overall uncertainty of the state is represented by the RSO's covariance, which grows as time elapses. Maintaining a low covariance on all RSOs by conducting frequent observations is thus paramount to maintaining SDA. Preferably, "observations should be taken at different positions on a satellite's orbital path [. . . ] ideally, cover[ing] the full 360 degrees of an orbit" but this is unrealistic given resource constraints (USSTRATCOM, 2004b). Observations are instead planned using scheduling theory algorithms and analyst judgment.

Near-Earth RSOs are traditionally tracked using ground-based radars, while Deep Space RSOs are monitored using ground-based telescopes. Space-based observation platforms are capable of tracking RSOs in any regime. Optical observations using NFOV telescopes typically use the sidereal collection mode, in which the telescope focuses on the background stars at the suspected location of the RSO and collect light over a short time interval, thus 


\section{JDAL 5,1}

80
Figure 1.

Optical collection chain (the red RSO is collected by the blue telescopes only if SNR constraints, determined by supporting parameters, are fulfilled) forming a streak in the data from which the endpoints are used to generate two observations (Campbell et al., 2018). Multiple observations on an RSO are ultimately combined in a process called orbit determination in which RSO state and covariance information is updated. This information is used to propagate an RSO's position forward or backward in time to conduct the SDA and STM missions.

Figure 1 outlines the typical optical collection chain for a ground-based telescope, which are traditionally limited to nighttime collections because of daytime brightness. Ultimately, a sufficient signal-to-noise ratio (SNR) at the telescope's sensor array is required for a successful collection. Howell's (2006) and Shell's (2010) equations are useful in defining the radiometry of the problem. Schmunk's approach estimates the RSO's light at the telescope given a known standard visual magnitude $\left(M_{v}\right)$ (Schmunk, 2008). A general background brightness of 19 to $21 M_{v} / \operatorname{arcsec}^{2}$ may be assumed for dedicated sites on clear moonless nights while Krisciunas and Shaffer's work informs simulating lunar brightness (Krisciunas and Schaefer, 1991). Tools such as the AFIT-developed Laser Environmental Effects Definition and Reference (LEEDR) routine model atmospheric conditions (Fiorino et al., 2008). Clouds may be modeled stochastically based on known empirical data sets.

\section{Current USG SDA processes, challenges and impetus for change}

RSO cataloging is performed by several ground and space-based sensors. Three GroundBased Electro-Optical Deep Space Surveillance (GEODSS) sites, each with three $1 \mathrm{~m}$ telescopes in New Mexico, Maui and Diego Garcia comprise the major Deep Space ground collection systems. An improved future system, the Space Surveillance Telescope (SST), is planned to begin operations from Australia in 2022. Three space-based platforms - SpaceBased Space Surveillance (SBSS), Operationally Responsive Space 5 (ORS-5) and Sapphire also contribute to the mission.

RSO collections are split between a tasking and scheduling approach in which various sensors are tasked to collect on RSOs of interest in a centrally managed process, but actual observations are scheduled by the sensors. Data is processed through the Space Defense Operations Center (SPADOC) and Correlation, Analysis and Verification of Ephemerides

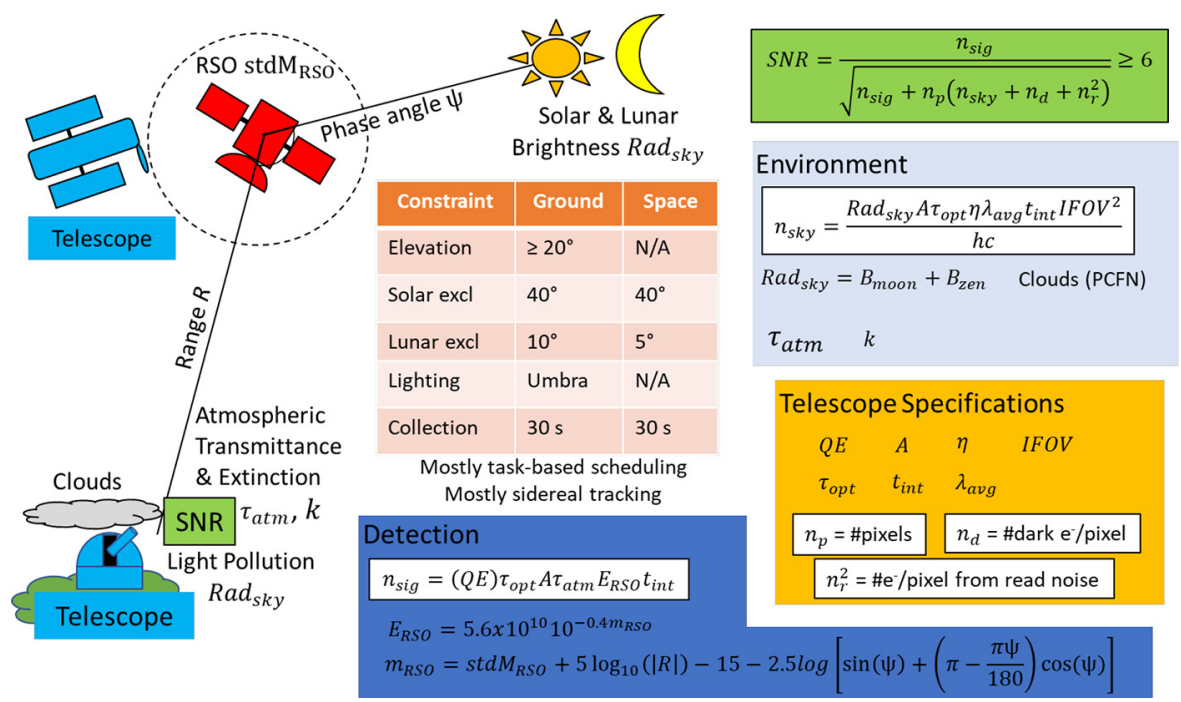


Network (CAVENet) networks. These systems represent the first challenge to maintaining SDA. Developed as far back as the 1980s, these systems and processes have long approached end of life, described by the Air Force Space Command (AFSPC) Commander in 2017 as "limping across the finish line until we can get [a new system] up" (Raymond, 2017). Processing capabilities are estimated to be $2-3$ orders of magnitude below a mid-2000s Web server (Weeden, 2012). The most recent upgrade was canceled in 2019 as replacing the system without impacting critical operations proved difficult, as well as determining how to incorporate a major influx of new data (Clark, 2019). The USG is challenged from a awareness technological perspective to maintain its own capabilities.

From the amalgamated data, RSO state information is re-calculated on demand for highinterest RSOs every eight hours for Near-Earth RSOs and every $12 \mathrm{~h}$ for Deep Space RSOs (Lal et al., 2018). Positional information is distributed in the form of Two Line Element sets (TLEs), without covariance information, on the public Space-Track database by the 18th Space Control Squadron (18 SPCS). However, this information is insufficient for many modern users.

The final two challenges impacting future SDA needs are the expansion of space utilization and the need to address new threats. Analysts project a growth in space services, with deployments of large constellations of hundreds of satellites already underway. This has in part been spurred by more frequent, affordable launch opportunities with technological advances in satellite capabilities. Increased adversarial threats are also prompting additional SDA needs.

Recognizing these problems, Space Policy Directive 3 (SPD-3) was released by the President in 2018 (Trump, 2018). This call to action asserts that "as the number of space objects increases... [the current] limited traffic management activity and architecture will become inadequate" and directs executive departments to pursue: improvements in observational data, algorithms and models; developing new hardware and software to support data processing and observations; mitigating the effect of orbital debris; improving SDA data interoperability; and enabling greater data sharing. Agencies were also directed to improve SDA coverage and accuracy by seeking to minimize deficiencies in SDA capability, "particularly coverage in regions with limited sensor availability and sensitivity in detection of small debris" through data sharing, data purchase, or the provision of new sensors; developing better tracking capabilities; and developing the standards and protocols for creation of an open architecture data repository.

Air Force Space Command Instruction 10-610 (AFSPCI 10-610), Space Situational Awareness Metric Data Integration Guidelines for Nontraditional Sensors, provides a defense-focused response to SPD-3 (AFSPC, 2019). The guidance outlines how to explore using nontraditional capabilities, stating that the "intent is to improve SDA through the utilization of a wide variety of sensor data and ephemeris data, of varying fidelity and accuracy, from an array of USG, non-DoD, commercial, civil and foreign data providers." It also emphasizes that "the quality and compatibility criteria for new data sources should be set as broadly as possible." Data challenges have been partially addressed by the creation of the Nontraditional Data Pre-Processor (NDPP) which allows ingesting data from new sources. The crux of this research lies on formulating an objective study to analyze these alternatives.

\section{Extant nontraditional optical sensors}

There are a plethora of telescopes with the ability of contribute to the USG's SDA needs. An example of a civil sensor whose contributions may be easy incorporated is the National Aeronautics and Space Administration's (NASA's) $1.3 \mathrm{~m}$ Meter-Class Autonomous 


\section{JDAL 5,1}

Telescope (MCAT) sensor on Ascension Island. Used to statistically characterize orbital debris, it has already been proposed for use as a contributing sensor to the SSN (Lederer et al., 2013). Additionally, Lal et al. found a "recent development has been the repurposing of existing sensors previously used for astronomy and other scientific research "for SDA purposes (Lal et al., 2018). More broadly, SDA data gathered incidentally by sensors performing related missions such as astronomy may be useful. Bellows demonstrated that RSO positional updates can be obtained using data from RSO streaks gathered serendipitously by astronomical telescopes, citing the Panoramic Survey Telescope and Rapid Response System (Pan-STARRS) and the Large Synoptic Survey Telescope (LSST) as capable of providing advantages to DoD (Bellows, 2015).

Multiple worldwide commercial networks rivaling DoD's coverage at the expense of lower fidelity now exist. These capabilities came about in part by "perceptions related to lack of transparency with DoD data" and improvements in optical sensing technology with a reduction in parts cost (Lal et al., 2018). Major optical Deep Space cataloging companies include Analytical Graphics Incorporated (AGI), ExoAnalytic Solutions and the Numerica Corporation. As an example of commercial capabilities, ExoAnalytic offers an extensive list of services which includes simply purchasing its data or pursuing a turn-key package of one 14 in telescope with $1^{\circ} \mathrm{NFOV}$ capable of observing RSOs down to $18.5 M_{v}$ for $\$ 500 \mathrm{~K}$ (ExoAnalytic Solutions, 2020).

\section{Related studies}

No study has considered improving the USG's SDA cataloging in the manner proposed herein; however, related studies have guided this approach. Moomey successfully translated a Commander's Intent to improve SDA cataloging into the simulation of a worldwide network of small rate-tracking commercial telescopes through the systems engineering process, demonstrating that adding 60 telescopes at each of five worldwide sites could allow up to $2 \mathrm{~h}$ of extra time per night on each GEODSS telescope (Moomey, 2015). Raley et al. highlighted the Defense Advanced Research Project Agency's (DARPA's) OrbitOutlook effort, demonstrating that data from Space-Track and multiple civil, commercial, academic and hobbyist providers could be combined to improve overall SDA goals while overcoming data surety concerns (Raley et al., 2016). Richard et al. developed a framework to design survivable radar satellites via Multi-Attribute Tradespace Exploration (MATE) using decision-maker input to identify attributes and weightings, propose design variables and realistic architectures and use modeling and simulation (M\&S) to find Pareto-efficient architectures based on cost and utility (Richard et al., 2009). Limitations of Moomey and Raley et al. include failing to optimize the AN tradespace while Richard et al. omitted a large-scale M\&S because of computational limitations.

Several studies exploring the development of a new, alternative network to the SSN are pertinent. Ackermann et al.'s, 2015 work coupled modeling and simulation with expert knowledge of telescope siting constraints to design a network to outperform the SSN's Deep Space tracking capabilities (Ackermann et al., 2015). Colombi et al. sought to create the optimal network of SDA cataloging sensors from a large tradespace of ground and space-based telescopes by evaluating multiple architectures using MOO powered by a physics-based M\&S of a nightly collection scenario (Colombi et al., 2018). Work by Bateman et al. (2018) and Felten et al. (2018) extended Colombi et al.'s work with an improved methodology, M\&S and additional sensors. Although these studies were comprehensive and insightful, they neglected the reality that a clean-slate approach to USG tracking is unrealistic in the current fiscal environment.

The last area of study concerns inferring likely AN requirements and performance measures. USG SDA and SSN requirement documents are felt to best inform this 
consideration; however, no open-source documents on these subjects are found in literature so proxies are assumed. Moomey's end-to-end system engineering approach with mission requirements and measures best illuminate the end-to-end requirements process and desired outcomes (Moomey, 2015). Daw and Hejduk's, 1999 study on SSN operational capabilities identified three target areas with associated performance measures which lend themselves to this study: suitability, including accessibility and connectivity; effectiveness, including coverage, capacity, responsively and detectability; and performance, including timeliness and quality (Daw and Hejduk, 1999). Finally, AFSPCI 10-610 outlines desirable sensor criteria in Military Utility Assessment (MUA) criteria which are considered. These include accuracy, capacity, sensitivity, field of regard/orbital coverage, search rate, tasking responsiveness, unique capabilities, availability, reliability and cost (AFSPC, 2019).

\section{Methodology}

Figure 2 illustrates the methodology. The SPO purchase decision discussed in the Introduction section is addressed using Crawley et al.'s approach, which requires modeling and simulating architectural permutations, assessing them based on performance measures and using Pareto optimality to evaluate alternatives. Multiple days are assessed using methods similar to those of Epoch-Era analysis.

\section{Develop representative sites and sensors}

The nine $1 \mathrm{~m}$ GEODSS telescopes are chosen to form the baseline USG architecture; spacebased capabilities are neglected in this study. Three separate hypothetical commercial companies with several $0.3-0.8 \mathrm{~m}$ telescopes are developed based on open-source literature review. The GEODSS and commercial sensors are assumed to operate in one centrally tasked, centrally scheduled manner performing the core USG SDA mission because of exclusive purchase rights. A sensor similar to NASA's $1.3 \mathrm{~m}$ MCAT on Ascension Island is chosen to be a civil contributor, providing information mostly on debris. A hypothetical large-aperture Wide Field of View (WFOV) telescope, the Brazil-Internacional Gigante Global Observatorio (BIGGO), is developed as a scientific contributor which serendipitously collects on RSOs while performing an astronomy mission at a fixed sky position. All telescopes with the exception of BIGGO are assumed NFOV and use a task-based search method.

\begin{tabular}{|c|c|c|}
\hline $\begin{array}{l}\text { Scenario } \\
\text { - USG SPO has \$25M to incorporate } \\
\text { data from new sensors into SSN } \\
\text { - Buy from } 3 \text { commercial providers \& } \\
\text { take free data from civil and scientific } \\
\text { - Find best choice for GEO cataloging }\end{array}$ & $\begin{array}{l}\text { Develop Representative Sites/Sensors } \\
\text { - USG: } 9 \times 1 \mathrm{~m} \text { GEODSS } \\
\text { - Commercial: } 56 \times 0.3-0.8 \mathrm{~m} \text { NFOV } \\
\text { - Civil: } 1.3 \mathrm{~m} \text { NASA/AFRL MCAT } \\
\text { - Scientific: } 2 \mathrm{~m} \text { WFOV in Brazil }\end{array}$ & $\begin{array}{l}\quad \text { Translate Needs } \rightarrow \text { Measures } \\
\text { - Lit review \& brainstorming used to } \\
\text { generate likely AN needs/requirements } \\
\text { - Three measures best capture needs: } \\
\text {-- Avg Capacity } \\
\text {-- Avg Max Time Unobserved } \\
\text {-- Coverage }\end{array}$ \\
\hline 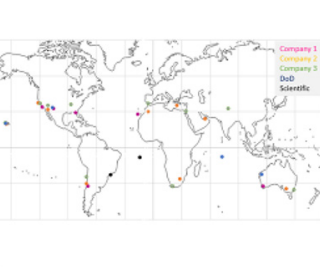 & $\begin{array}{l}\text { Telescopes/Cameras } \\
\text { OSR Lg Astro } 600 \mathrm{~F} 3.8 \mathrm{w} / \mathrm{FLI} \text { ML09000 } \\
\text { Takahashi FET-300 w/FLI ML09000 } \\
\text { OSR Lg Astro } 800 \mathrm{F5} \text { w/Apogee ALTA F } 4320 \\
\text { ASA Astro } 16 \mathrm{~N} \text { Reduced w/FLI ML09000 } \\
\text { Takahashi C-400 w/Apogee ALTA F4320 } \\
\text { ASA Astro } 16 \mathrm{~N} \text { Reduced w/FLI ML16803 } \\
\text { OSR Lg Astro } 600 \mathrm{~F} 5 \text { w/Apogee ALTA F4320 } \\
\text { BIGGO w/Spectral Instrument } \\
\text { MCAT w/Spectral Instrument } \\
\text { GEODSS w/Sarnoff MIT/LL CCID-16 }\end{array}$ & $\begin{array}{l}\quad \text { Develop M\&S } \\
\text { - Goal: M\&S ANs using } 24 \text { hr collection } \\
\text { scenario on } 2 \text { days, calculate measures } \\
\& \text { ID 'best' via optimization routine } \\
\text { - Get GEO RSO TLEs and Standard Mv } \\
\text { and assign Category } 1-5 \text { importance } \\
\text { - Propagate RSOs on both days \& get } \\
\text { Sensor-RSO access times } \\
\text { - Break collections into } 30 \text { s windows, } \\
\text { model RSO SNR \& keep only SNR } \geq 6\end{array}$ \\
\hline $\begin{array}{l}\text { Assess } \\
\text { - } \sim 100 \text { archs are in Top } 10 \% \text { both days } \\
\text { - Apply additional considerations to } \\
\text { identify } \sim 15 \text { high-performing options } \\
\text { - Decision-maker empowered with } \\
\text { multiple best ways to improve SDA }\end{array}$ & $\begin{array}{l}\text { Run M\&S } \\
\text { - Do } 5 \text { NSGA-II trials for } 100 \text { archs/50 gens } \\
\text { - Run scheduler on each arch, calculate } \\
\text { measures, solve optimization problem } \\
\text { - Architectures advance using selection, } \\
\text { binary crossover \& mutation }\end{array}$ & $\begin{array}{l}\text { Schedulers } \\
\text { - GEODSS/commercial: en masse } \\
\text {-- Very dim > old obs'd RSOs > Cat 1-5 } \\
\text {-- Bins sorted by RSO w/fewest accesses } \\
\text { - Civil tasks on debris for first/last obs hr } \\
\text { - Scientific gets serendipitous in FOV }\end{array}$ \\
\hline
\end{tabular}

Space domain awareness

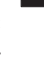


JDAL 5,1

Hypothetical locations, sensor specifications and business cases are developed for the commercial networks based on review of open-source material and artistic license. Realworld telescope and camera specifications are used in the simulation. Table 1 summarizes the business cases.

\section{Translate needs into measures}

Moomey's systems engineering approach is applied to identify possible AN requirements, from which many performance measures are derived. The measures are compared to those identified in literature and three are deemed common and practical for this study. Their algebraic formulations are covered in a subsequent subsection:

- Average capacity: capacity is defined as the number of times a particular RSO is observed by sensors in the architecture; the average value amongst all RSOs is computed.

- Average maximum time unobserved: time unobserved is defined as the maximum time an RSO goes unobserved during the scenario; the average value amongst all RSOs is computed.

- Coverage: coverage represents the ability of sensors to track regions of the GEO belt with managed redundancy. The $\pm 15^{\circ}$ latitude region defining the GEO belt is broken into $1^{\circ} \times 1^{\circ}$ bins. Each bin is assigned a value based on the number of sensors capable of viewing it and a diminishing returns formula is used to discourage an architecture with all sensors in one location. The coverage measure is computed by summing the values in all bins.

The approach to calculating these measures has been refined over previous iterations of this work. High coverage was found to be easily achievable when cast as the ratio of observed RSOs to those simulated. A weighted geometric value is proposed to better capture viewability of RSOs while encouraging geographically dispersed, redundant capabilities. Average Maximum Time Unobserved is proposed to better report the temporal frequency of observations. Previously, average and maximum revisit times were used, unexpectedly causing large variations when adding new observations further in time from a core group and unfairly penalizing any RSO receiving an extra observation. The current approach minimizes this occurrence.

Company

(\#Sensors) Business case

$1(12) \quad$ Limit number of sites/sensors and use mostly $0.3 \mathrm{~m}$ telescopes $(\$ 1 \mathrm{~m})$

- Offset high costs w/expensive, exquisite capabilities in high-interest locations

- Use 0.6 m in Teide, Canary Islands ( $\$ 1.5 \mathrm{~m}) ; 0.6 \mathrm{~m}$ in El Leoncito, Argentina ( $\$ 1.5 \mathrm{~m})$; and $0.8 \mathrm{~m}$ in Perth, Australia $(\$ 2 \mathrm{~m})$

- A discount of $\$ 0.2 \mathrm{~m}$ is awarded if either all three large telescopes or all telescopes in the network are picked

2 (21) - Mix $0.3 \mathrm{~m}$ and $0.4 \mathrm{~m}$ with different FOVs

Table 1.

Hypothetical company capabilities and business cases
$3(23)$

- Charge more for $0.4 \mathrm{~m}(\$ 0.75 \mathrm{~m})$ than the $0.3 \mathrm{~m}(\$ 0.50 \mathrm{~m})$

- Charge $\$ 0.25 \mathrm{~m}$ extra for any site in the southern hemisphere

- Charge a low cost for a standard sensor $(0.40 \mathrm{~m}, \$ 0.50 \mathrm{~m})$

- Charge more for its locations in Israel and India (0.6 m in India)

- Charge additional $\$ 0.2 \mathrm{~m}$ if multiple sensors are used at one site 
Figure 3 illustrates the M\&S framework, which requires various supporting tasks prior to running the actual assessment. Colombi et al.'s basic M\&S methodology is adapted and modified. Unless otherwise stated, all tasks are completed using Python with its Pandas database and NumPy numerical libraries.

First, 954 GEO RSOs and their TLEs are pulled from Space-Track on 7 Mar 2020. RSO names are fused with owner, status as active or debris, mission and standard Mv data from Celestrak, Gunter's space database and Calsky. The RSOs are binned into five categories, awareness with Category 1 being highest importance, based on analyst judgment and in proportion to percentages suggested by 18 SPCS in similar work (Dararutana, 2019).

Site latitudes and longitudes are stored in a separate file. LEEDR is used to estimate atmospheric transmittance and extinction values at all sites during summer and winter nights. A choice is made to handle clouds by merely blacking out a sensor for an entire night if a randomly generated number exceeds a threshold. This threshold, the Probability of Cloud-Free Night (PCFN), is developed using data from the 14th Weather Squadron (14 WS). Up to 30 years of sky coverage data from hourly meteorological reports is analyzed to estimate the average nightly cloud coverage at each site for every calendar month. A cloud-free night is assumed as a night with average sky coverage less than or equal to five oktas on a zero to eight scale. The proportion of cloud-free nights at each site for a given calendar month garners the appropriate PCFN.

Because weather is stochastic, several Monte Carlo simulations with different cloud conditions are designed. Each Monte Carlo is represented by a string of binary bits, where each bit represents a site, with one indicating cloud-free conditions and zero too cloudy to collect. The determination for a one or zero is based on the comparison of random-number generator (RNG) values to PCFN thresholds. A total of 200 weather simulations are deemed sufficient after a small set of architectures is tested using 10,000 Monte Carlos. Oberle's percent error-based approach using the Central Limit Theorem states that the minimum number of simulations may be found by taking a sample with a large number of Monte Carlos, assuming the sample standard deviation and averages are close to the those of the population and calculating:

$$
m=\left(z_{\alpha / 2} \frac{100 s}{\varepsilon Y_{a v g}}\right)^{2}
$$

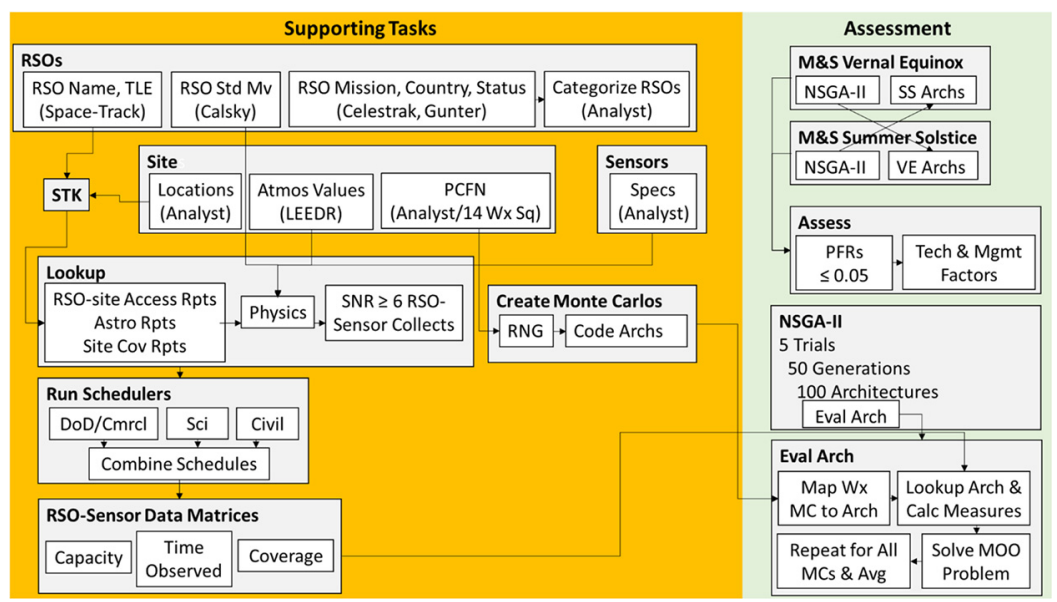

Figure 3.

M\&S framework (architectures are assessed on two different days based on information generated by various supporting tasks) 


\section{JDAL 5,1}

for each measure, where $z_{\alpha / 2}$ is the $z$ statistic, $\varepsilon$ is the percent error, $s$ is the sample standard deviation and $Y_{\text {avg }}$ is the sample average (Oberle, 2015). For a 95\% confidence interval $(z=1.96)$ and a two percent error $(\varepsilon=2)$, analysis of the set show that at least 90 simulations are necessary. A second calculation to illustrate the percent change of the measures' averages as the number of Monte Carlos is increased shows the point at which the percent change is consistently less than $0.5 \%$ occurs with 200 simulations. A total of 200 simulations are felt to be appropriate.

Two STK scenarios are created containing all sites and RSOs; one for Vernal Equinox and one for Summer Solstice. These dates are chosen for evaluation because of their poor collection conditions; GEO RSOs experience a 1-h eclipse prohibiting detection on the former, while the shortest nights in the northern hemisphere occur on the latter (Colombi et al., 2018). On each date, RSOs are propagated forward in time over a $24 \mathrm{~h}$ period using the Standard General Perturbations 4 (SGP4) algorithm, which neglects covariance information and assumes a perfectly known RSO position. Sites are constrained to operate only in umbra and track RSOs at an elevation $\geq 20^{\circ}$, a solar exclusion angle of $40^{\circ}$, and a lunar exclusion angle of $10^{\circ}$. Note that sensor parameters are modeled later in STK.

An assumption is made that all sensor-RSO collections require $30 \mathrm{~s}$ to settle, take observations and slew to the next target. This allows the $24 \mathrm{~h}$ day to be broken into 2,880 finite intervals, greatly simplifying the scheduling discussed later. STK's astrodynamics routines are then used to output various reports in $30 \mathrm{~s}$ intervals, including a list of all possible site-RSO accesses, an array of $1^{\circ} \times 1^{\circ}$ coverage areas for sites and various quantities required for lunar brightness modeling.

All data sources are then combined to determine possible scheduling opportunities. SiteRSO accesses are expanded to include all sensors at each site. All sites are assumed to operate with a clear moonless night background radiance of $21 M_{v} / \operatorname{arcsec}^{2}$ while lunar and RSO intensity are estimated and incorporated separately via the approach previously outlined. Only sensor-RSO scheduling opportunities with SNR $\geq 6$ are kept.

Three separate schedulers are run: combined GEODSS/Commercial, MCAT and BIGGO. For all three, a simplification is levied that the scheduler be run only once up front and observations from particular sensors be taken from a master list when doing architectural evaluations. This sidesteps running the scheduler on every architectural evaluation, which is time-prohibitive.

The GEODSS/Commercial scheduler is custom-designed to quickly schedule a prioritized list of RSOs for geometrically and temporally dispersed collections. Based on data from Space-Track and Calsky as well as analyst judgment, RSOs are sorted in a prioritized list in the following manner: extremely dim (approximately 20 RSOs), very old TLEs (approximately $20 \mathrm{RSOs}$, only on first scheduling pass) and Category 1-5 observations. Within each grouping all RSOs are sorted by number of available accesses to encourage less-available RSOs to be scheduled first. The RSOs are scheduled via a loop until no sensorRSO collections remain. Each RSO is scheduled for a maximum of 20 observations at a time so as to prevent higher-ranked RSOs from receiving all possible collections. The 20 observations are spread evenly amongst the available sensors based on geography, then randomly assigned timeslots; this serves as a surrogate for collecting along multiple parts of the orbit, thus reducing RSO covariance uncertainty. At the end of the loop, the RSOs are sorted again in the same manner. This process of scheduling GEODSS/commercial observations requires around one hour on an Intel i5 Sandy Bridge laptop with two cores/four threads operating at $2.5 \mathrm{GHz}$ with $8 \mathrm{~Gb}$ of Random Access Memory (RAM).

MCAT is scheduled using a similar routine. Because of its various missions, it is set to track only at the first and last hour of the night. GEO debris is prioritized; when complete, 
active satellites are collected. BIGGO is not scheduled per say; it is assumed to point at a fixed azimuth and elevation for the entire scenario performing an astronomical mission. Any RSOs passing within $\pm 2^{\circ}$ of this position of sufficient brightness is assumed to be serendipitously collected. This results in several GEO RSOs being collected for several hours. In lieu of reporting all data on these RSOs, only the first and last opportunities for each are returned as contributions.

Model and simulation: assessment

Space domain awareness

The simulation is run for both days, each day using Deb's Non-Sorted Genetic Algorithm II (NSGA-II) heuristic routine in the Python Inspyred module (Garrett, 2012). All architectures assessed on one day are then evaluated under conditions for the other to permit a fair comparison. Using Python's Multiprocessing routine to parallelize the evaluation of architectures in each generation, the simulation completes in $24 \mathrm{~h}$ on an Intel Xeon Sandy Bridge Workstation with 16 cores and 32 threads running at $2 \mathrm{GHz}$ with $56 \mathrm{~Gb}$ of RAM.

NSGA-II is used as follows. As the nine GEODSS sites, MCAT and BIGGO are always used in the scenario, the only variable sensors in the architecture are the set of 56 commercial telescopes. An architecture is thus represented as a 56-gene binary chromosome, where the choice to use the sensor is represented by one or zero, for a total of $10^{17}$ possible AN permutations. For each day, five separate NSGA-II trials of 50 generations and 100 architectures each are evaluated. Randomly selected architectures meeting the cost constraint are used in the first generation. Each architecture is evaluated for the 200 weather conditions, and results averaged together for final architecture performance measures before crossover, mutation and selection occurs. All architecture costs and performance measures are saved for post-processing.

Each architecture evaluation solves the following pseudo optimization problem:

$$
\begin{gathered}
\text { Maximize } c=\left\{\begin{array}{cc}
\frac{1}{m} \sum_{j=1}^{m}\left(\sum_{b=1}^{b}\left(\sum_{s=1}^{s} \frac{1}{s}\right)\right), & d \leq 25 \\
\text { penalty, } & d>25
\end{array}\right. \\
\text { Maximize } \bar{p}=\left\{\begin{array}{cc}
\frac{1}{m} \sum_{j=1}^{m}\left(\frac{1}{n} \sum_{i=1}^{n} r_{i}\right), & d \leq 25 \\
\text { penalty, } & d>25
\end{array}\right. \\
\text { Minimize } \bar{l}=\left\{\begin{array}{cc}
\frac{1}{m} \sum_{j=1}^{m}\left(\frac{1}{n} \sum_{i=1}^{n} \max \left(\Delta t_{0} \ldots \Delta t_{f}\right)\right), & d \leq 25 \\
\text { penalty, } & d>25
\end{array}\right.
\end{gathered}
$$

where $c$ is coverage, $s$ is the sensor capable of observing the $1^{\circ} \times 1^{\circ}$ bin, $b$ represents all bins covering the GEO belt, $m$ is the number of Monte Carlos, $\bar{p}$ is average capacity, $r_{i}$ is the observed RSO, $n$ is the total number of RSOs observed by the architecture, $\bar{l}$ is the average maximum time unobserved, and $\Delta t$ represents all unobserved durations for each RSO. A large penalty is applied if the $\$ 25 \mathrm{M}$ cost constraint is exceeded, making the value unfavorable to the optimizer. The underlying values are computed by conducting operations on efficient arrays based on the list of scheduled observations. 


\section{JDAL 5,1}

88

\section{Findings}

All architectures and top $10 \%$ performers

In total, 50,000 architectures are evaluated in total, of which $68 \%$ are feasible because of the cost constraint. For each date, the feasible architectures' measures are sorted using a modification to Blank and Deb's fast non-dominated sort routine from PyMoo (Blank and Deb, 2020). Architectures are assigned a Pareto Front Ratio (PFR) based on their relative ranking amongst the fronts, where one indicates architectures on the best front. Figure 4 illustrates results on Vernal Equinox, where the average maximum time unobserved and average capacity are plotted with a color spectrum for coverage. The highest performers are those in the bottom right colored purple. Results for Summer Solstice are holistically similar.

Figure 5 plots individual architectures by PFRs on each day with a spectrum color-coding by cost, with higher cost approaching purple. A line representing architectures with equal PFRs on each day is also plotted for comparison. Architecture performance on both days is roughly proportional; however, deviations of up to $40 \%$ are observed. This illustrates the necessity of testing architectures on multiple dates. Cost is seen to increase as higher-performing architectures are encountered, which is intuitive as adding more sensors improves capability.

Top performers are those architectures with low PFRs on both dates; a determination is made that only the 187 architectures with PFRs $\leq 0.05$ on both days be considered in the rest of this analysis. These architectures cost a minimum of $\$ 23.5 \mathrm{~m} ; 81 \%$ cost at least $\$ 24.5 \mathrm{~m}$. Each architecture consists of 33-35 commercial sensors. In total, 16 specific sensors of the 56 available (29\%) are used in over $80 \%$ of the top performers, while two are used in less than $20 \%$. It is difficult to make sweeping conclusions about sensor performance in the larger problem as the architectures are only a small sample from the large trade space. Factors

Figure 4.

Architecture performance on vernal equinox; higher performers are on the bottom right

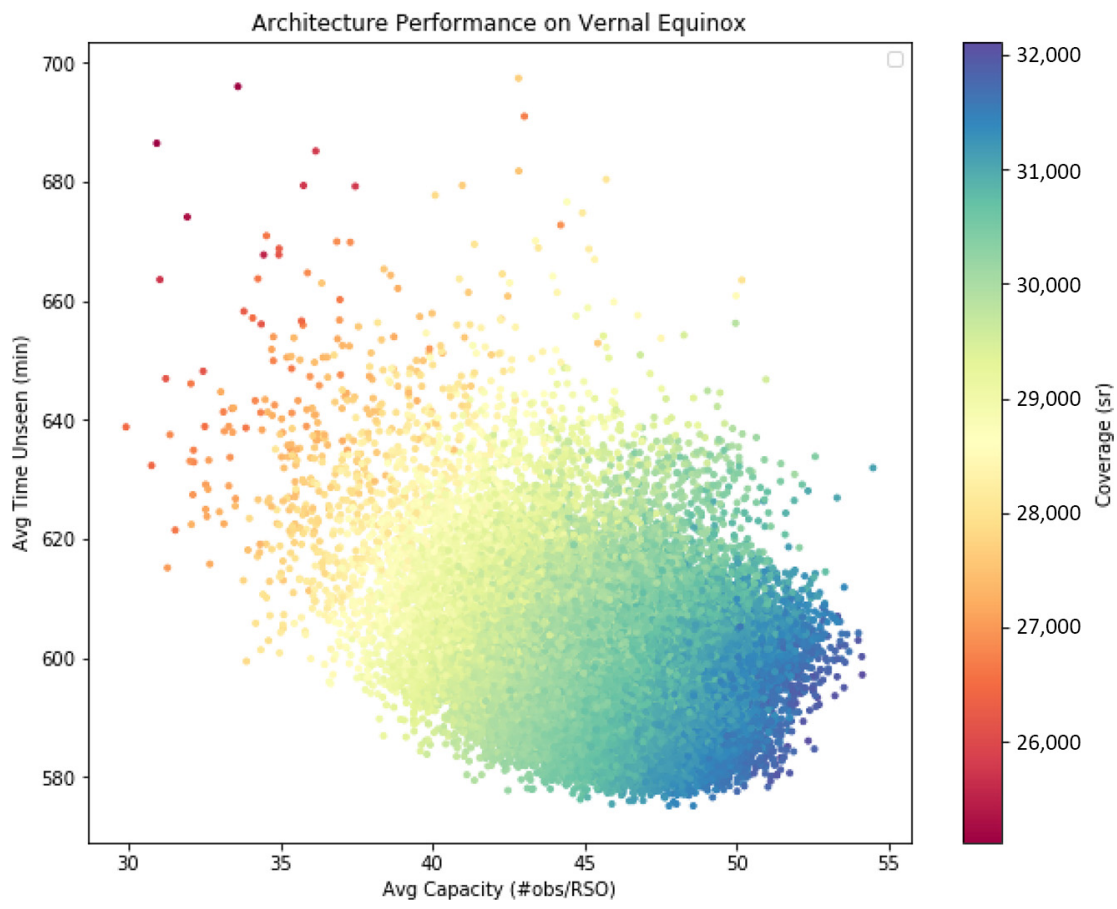




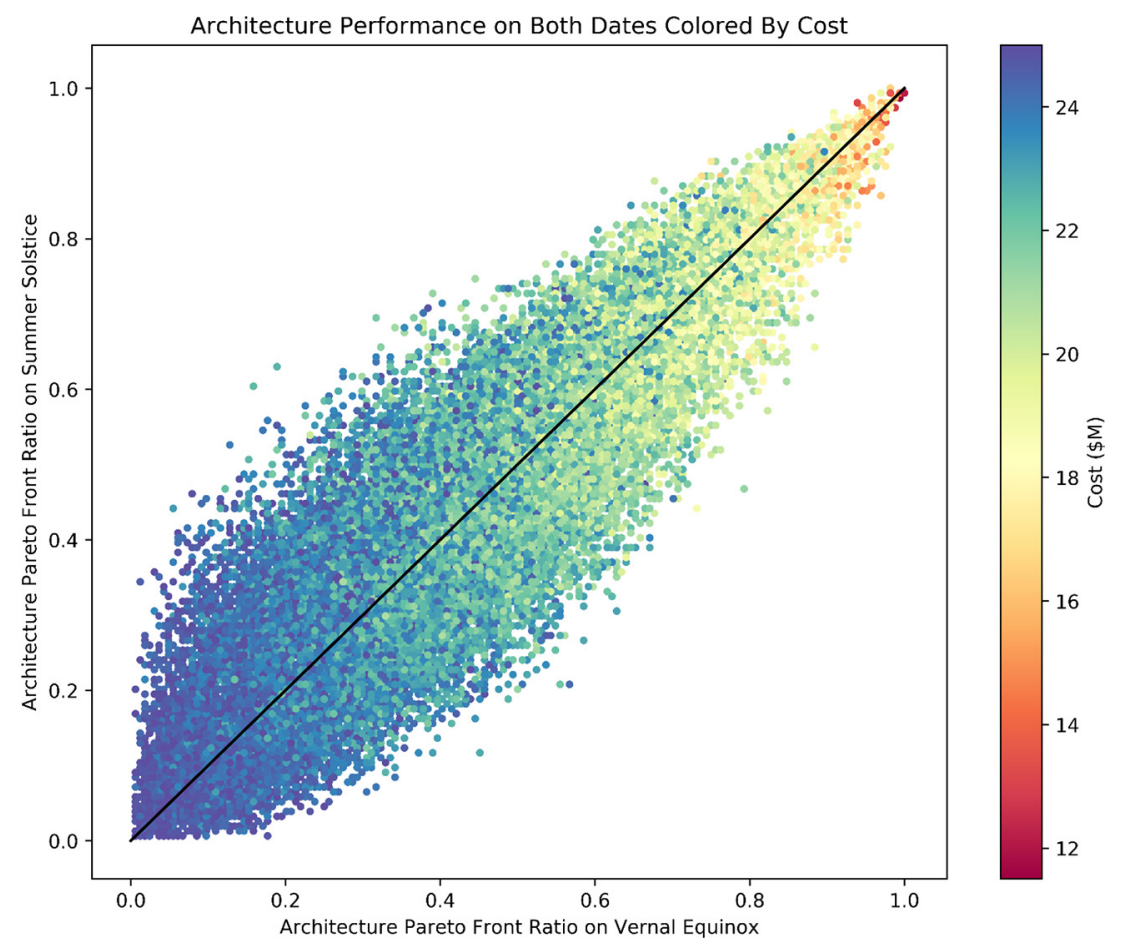

Space domain awareness

89

Figure 5.

Relative architecture performance on both days (higherperforming architectures appear in the bottom left; cost increases with increasing performance)

contributing to the selection results include weather conditions, the ability to improve geographic diversity of the GEO belt, and the availability of GEO RSOs over sensors.

Figure 6 illustrates the performance of the top architectures. Around 49-51 average observations per RSO at a 595-630 average maximum time unobserved is achieved with global, redundant coverage. Average Capacity and Average Maximum Time Unobserved are better on Vernal Equinox than Summer Solstice, which may be expected because of Summer Solstice's shorter nights which minimizes the number of collections. The differences in coverage are because of different cloud conditions during the different times of year.

\section{Best performers based on additional considerations}

Additional technical and managerial considerations may be applied to further reduce the decision space. This includes quantifying the realization of performance measures; pursuing minor cost savings and award fee equity; and considering sensor utilization per company, which is a proxy for business sustainability.

A simple indicator of better performance is found through the sum of PFRs measure, IPFRs, which sums each architecture's PFR on both days; lower values are more favorable. The Percent Unrealized (PU) metric measures how far each architecture's performance measure values are from the best possible amongst the top performers such that:

$$
P U=\frac{\mid \text { best }- \text { achieved } \mid}{\text { best }} \times 100
$$


\begin{tabular}{l} 
JDAL \\
5,1 \\
$\mathbf{9 0}$ \\
\hline
\end{tabular}

Figure 6.

Architecture performance variation on each date
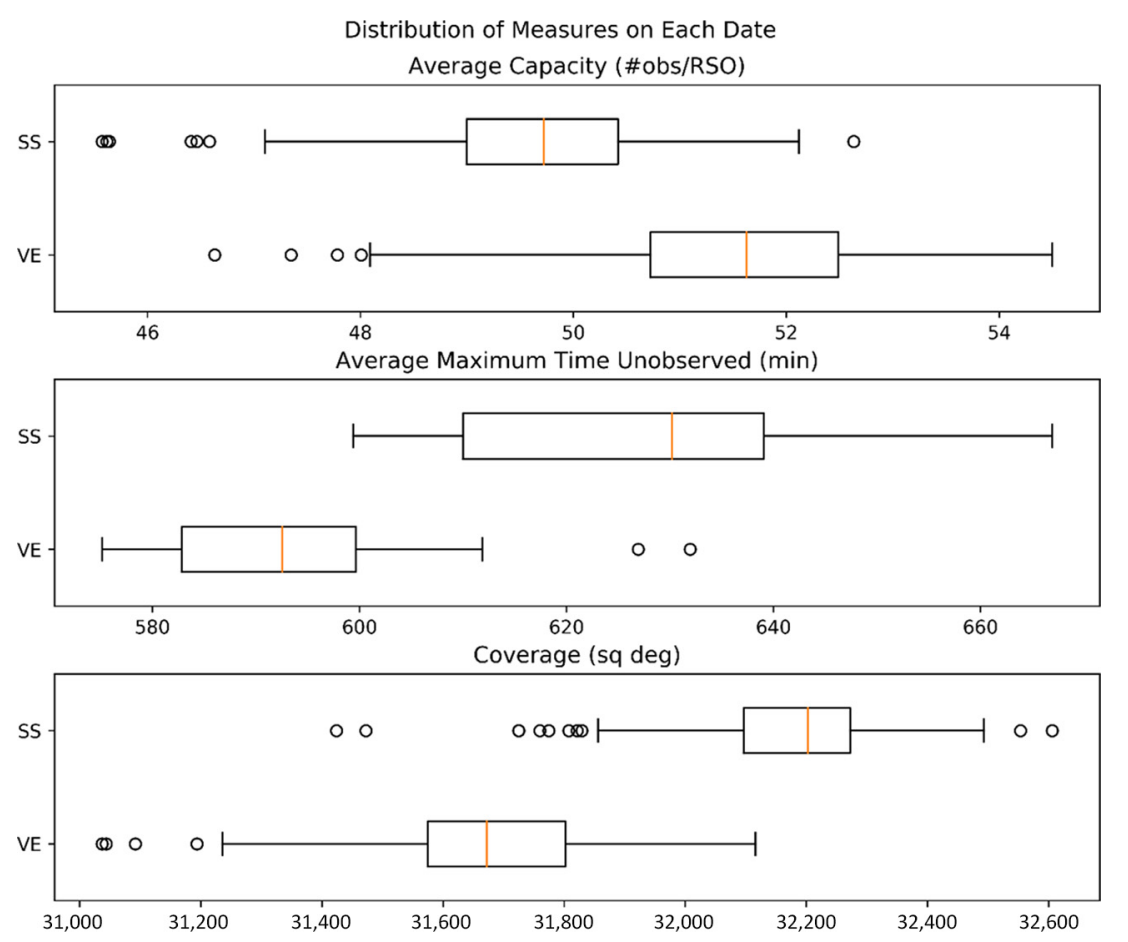

Architectural comparisons are drawn by creating a sum of sum of PU (SSPU) metric for all three metrics on both dates; lower sums indicate better relative performance.

The cost savings under the $\$ 25 \mathrm{~m}$ constraint is tabulated for consideration. Additionally, the equity in award fee is considered as it may help a decision-maker understand perceived fairness in competition. Finally, the sensor utilization equity metric is created and defined as the ratio of sensors from a company to those in the company's proposal. This is felt to be a proxy for a company's business case fulfillment. Taking the standard deviation of the three award fees $\sigma_{\text {awd }}$ and the standard deviation of the three sensor utilization equity values $\sigma_{u t i l}$, then looking for the lowest indicates those architectures with better value. Figure 7 illustrates the 19 architectures generated from these considerations which either fully

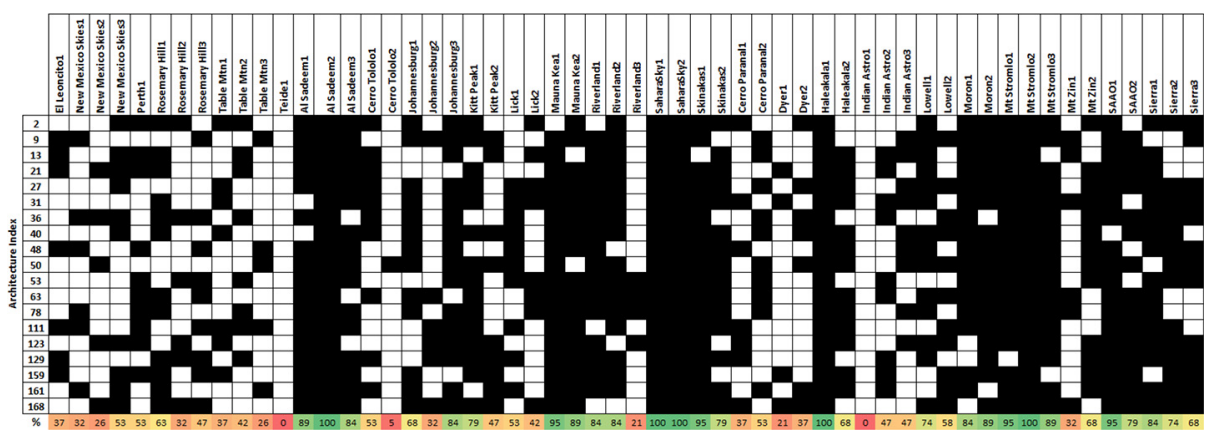

Figure 7.

Sensor use per architecture 
capitalize on one of these metrics or attempts to balance them in some manner. Thus, a further reduction in the decision space is achievable with modest additional considerations.

Five sensors are common to all architectures. Table 2 provides specific information on the best architectures for decision-maker action. The five metrics are color-coded columnwise based on the performance of all 187 top architectures using a red-yellow-green spectrum, where greener cells indicate better value for that metric.

\section{Comparison to baseline USG architecture}

Table 3 compares the baseline as-modeled $\mathrm{DoD}$ architecture of nine telescopes to the top performers of the AN. A three-fold improvement in capacity and coverage and a $16 \%$, or nearly two hour, reduction in maximum time unobserved can be achieved using the AN concept.

\section{Conclusion}

This study has addressed a potential solution and methodology to improve the USG's SDA cataloging dilemma. A hypothetical scenario in which nontraditional sensor contributions are added to the SDA architecture was developed and resolved via the system architecting

\begin{tabular}{rllllll}
\hline Arch & Savings $(\$ \mathrm{M})$ & $\sigma_{\text {awd }}$ & $\Sigma$ PFRs & SSPU & $\sigma_{\text {util }}$ & Notes \\
\hline 2 & 0.4 & 3.660 & 0.082 & 22.045 & 26.314 & Very good $\sigma_{\text {awd }}$ \\
9 & 1.45 & 4.003 & 0.081 & 23.623 & 27.658 & Very good savings \\
13 & 0.25 & 3.739 & 0.058 & 27.705 & 25.066 & Best $\sigma_{\text {util }}$ \\
21 & 0.45 & 3.573 & 0.069 & 23.624 & 25.370 & Best $\sigma_{\text {awd }}$ Very good $\sigma_{\text {util }}$ \\
27 & 0.55 & 5.161 & 0.013 & 13.231 & 36.133 & Best $\Sigma$ PFRs, Best SSPU \\
31 & 0.05 & 5.310 & 0.013 & 18.044 & 34.954 & Best $\Sigma$ PFRs \\
36 & 0.4 & 3.632 & 0.082 & 23.640 & 26.879 & Very good $\sigma_{\text {awd }}$ \\
40 & 1.25 & 4.570 & 0.069 & 23.399 & 31.543 & Good savings \\
48 & 0.7 & 3.653 & 0.081 & 22.317 & 25.977 & Very good $\sigma_{\text {awd }}$ \\
50 & 1.55 & 4.914 & 0.076 & 23.609 & 33.885 & Best savings \\
53 & 1.4 & 3.925 & 0.076 & 24.981 & 28.364 & Very good savings \\
63 & 0.25 & 4.463 & 0.019 & 17.133 & 30.661 & Very good $\Sigma$ PFRs \\
78 & 0.2 & 4.174 & 0.013 & 14.681 & 28.995 & Best $\Sigma$ PFRs, Very good SSPU \\
111 & 0 & 3.649 & 0.043 & 17.091 & 26.478 & Very good $\sigma_{\text {awd }}$ Good SSPU/ $\sigma_{\text {util }}$ \\
123 & 0.25 & 3.854 & 0.037 & 18.424 & 26.989 & Good $\sigma_{\text {awd }} / \Sigma$ PFRs/SSPU/ $\sigma_{\text {util }}$ \\
129 & 0.15 & 4.124 & 0.019 & 16.751 & 29.416 & Very good $\Sigma$ PFRs \\
159 & 0 & 3.649 & 0.056 & 19.799 & 25.370 & Very good $\sigma_{\text {awd }} / \sigma_{\text {util }}$ \\
161 & 0.45 & 4.385 & 0.019 & 16.529 & 30.566 & Very good $\Sigma P F R s$ \\
168 & 0.05 & 4.139 & 0.013 & 25.979 & 28.880 & Best $\Sigma$ PFRs \\
\hline
\end{tabular}

Space domain awareness

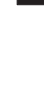


process. Performance measures were identified, extant capabilities were deduced and modeled, and physics-based simulations of multiple AN permutations were accomplished through the aid of optimization. The results show that top-performing architectures can provide a three-fold increase in coverage and capacity with a $16 \%$ reduction in maximum time unobserved by adding two contributing sensors and 33-35 geographically dispersed, fully taskable commercial sensors at a cost of around $\$ 25 \mathrm{~m}$.

To quote a renowned expert in the field, "all models are wrong, but some are useful" (Box, 1979). As Crawley et al's lower-fidelity architecting strategy was used in this effort, these results are perhaps best used to illustrate the potential benefits of this methodology in tradespace exploration. To that end, various improvements are anticipated in future work. First, the inclusion of the ground-based SST and various space-based DoD platforms, as well as a hypothetical fourth commercial company conducting space-based collections, will add to the reality of the problem. Additionally, improving the fidelity of the optical collection model will improve confidence in the intermediate calculations. Finally, treating RSO positions stochastically, instead of assuming perfect orbital knowledge as conducted herein, will better simulate the challenges of RSO collections during sensor scheduling and ultimately result in more realistic performance measure values.

\section{References}

Ackermann, M.R., Kiziah, R.R., Zimmer, P.C., McGraw, J.T. and Cox, D.D. (2015), “A systematic examination of ground-based and space-based approaches to optical detection and tracking of artificial satellites", Paper presented at the 30th Space Symposium Technical Track, 14 April, CO Springs, CO, available at: www.spacesymposium.org/wp-content/uploads/2017/10/M. Ackermann_31st_Space_Symposium_Tech_Track_paper.pdf (accessed 1 November 2020).

AFSPC (2019), "Air force space command instruction 10-610, space situational awareness metric data integration guidelines for Nontraditional sensors".

Bateman, M.G., Colombi, J.M., Cobb, R.G. and Meyer, D.W. (2018), "Exploring alternatives for geosynchronous orbit space situational awareness", Working paper, Air Force Institute of Technology, Wright-Patterson AFB, OH.

Bellows, C.T. (2015), "Leveraging external sensor data for enhanced space situational awareness", $\mathrm{PhD}$ Dissertation, Air Force Institute of Technology.

Blank, J. and Deb, K. (2020), "Pymoo: Multi-Objective optimization in python”, IEEE Access, Vol. 8, pp. 89497-89509.

Box, G.E.P. (1979), Robustness in the Strategy of Scientific Model Building, University of WI-Madison.

Campbell, T.S., Reddy, V., Larsen, J., Linares, R. and Furfaro, R. (2018), "Optical tracking of artificial earth satellites with COTS sensors", Paper presented at the Advanced Maui Optical Surveillance Conference, September, Kihei, HI, available at: https://amostech.com/TechnicalPapers/2018/ Poster/Campbell.pdf (accessed 1 November 2020).

Clark, C. (2019), "What about JMS? Air force reanimates 'old clunker' space tracking system”, Breaking Defense, 8 April, available at: https://breakingdefense.com/2019/04/what-about-jms-air-forcereanimates-old-clunker-space-tracking-system/ (accessed 1 November 2020).

Colombi, J.M., Stern, J.L., Wachtel, S.T., Meyer, D.W. and Cobb, R.G. (2018), "Multi-Objective parallel optimization of geosynchronous space situational awareness architectures", Journal of Spacecraft and Rockets, Vol. 55 No. 6, pp. 1453-1465.

Crawley, E., Cameron, B. and Selva, D. (2016), System Architecture: Strategy and Product Development for Complex Systems, Pearson, Hoboken, NJ.

Dararutana, K. (2019), "Comparison of novel heuristic and integer programming schedulers for the USAF space surveillance network”, Master's Thesis, Air Force Institute of Technology. 
Daw, R.S. and Hejduk, M.D. (1999), "Determining SSN operational system capability (SYSCAP)", in Spence, L. (Ed.), Proceedings of the 1999 Space Control Conference, Lincoln Laboratory, Lexington, MA, pp. 24-34.

Space domain awareness

ExoAnalytic Solutions (2020), "Products and services", available at: https:/exoanalytic.com/spacedomain-awareness/commercial-price-list/ (accessed 22 March 2020).

Felten, M.S., Colombi, J.M., Cobb, R.G. and Meyer, D. (2018), "Optimization of geosynchronous space situational awareness architectures using parallel computation", Paper presented at the Advanced Maui Optical Surveillance Conference, September, Kihei, HI, available at: https:// amostech.com/TechnicalPapers/2018/SSA/Felten.pdf (accessed 1 November 2020).

Fiorino, S.T., Bartell, R.J., Krizo, M.J., Caylor, G.L., Moore, K.P., Harris, T.R. and Cusumano, S.J. (2008), "A first principles atmospheric propagation and characterization tool: the laser environmental effects definition and reference (LEEDR)", in Korotkova, OL. (Ed.), Lasers and Applications in Science and Engineering: Atmospheric Propagation of Electromagnetic Waves II, SPIE, San Jose, $C A, 68780 B$.

Garrett, A. (2012), "Inspyred", available at: https://github.com/aarongarrett/inspyred (accessed 1 November 2020)

Howell, S.B. (2006), Handbook of CCD Astronomy, 2nd ed., Cambridge University Press, Cambridge.

Krisciunas, K. and Schaefer, B.E. (1991), "A model of the brightness of moonlight", Publications of the Astronomical Society of the Pacific, Vol. 103 No. 667, pp. 1033.

Lal, B., Balakrishnan, A., Caldwell, B.M., Buenconsejo, R.S. and Carioscia, S.A. (2018), "Global trends in space situational awareness (SSA) and space traffic management (STM)", Science and Technology Policy Institute, pp. 1-43.

Lederer, S.M., Stansbery, E.G., Cowardin, H.M., Hickson, P., Pace, L.F., Abercromby, K.J. and Kervin, P.W. (2013), "The NASA meter class autonomous telescope: Ascension island", Paper presented at the Advanced Maui Optical Surveillance Conference, September, Kihei, HI, available at: https:/ntrs. nasa.gov/citations/20130013162 (accessed 1 November 2020).

Maier, M.W. and Rechtin, E. (2010), The Art of Systems Architecting, 3rd ed., CRC Press, Boca Raton, FL.

Moomey, D. (2015), “A call to action: Aid geostationary space situational awareness with commercial telescopes”, Air and Space Power Journal, Vol. 29 No. 6, pp. 12-31.

Oberle, W. (2015), "Monte Carlo simulations: Number of iterations and accuracy”, United States Army Research Laboratory, Report ARL-TN-0684.

Parnell, G.S., Bresnick, T.A., Tani, S.N. and Johnson, E.R. (2013), Handbook of Decision Analysis, John Wiley and Sons, Hoboken, NJ.

Raley, J., Weisman, R., Chow, C., Czajkowski, M. and Sotzen, K. (2016), "OrbitOutlook: autonomous verification and validation of Nontraditional data for improved space situational awareness", Paper presented at the Advanced Maui Optical Surveillance Conference, September, Kihei, HI, available at: https://amostech.com/TechnicalPapers/2016/SSA-Algorithms/Raley.pdf (accessed 1 November 2020).

Raymond, J. (2017), "Speech, "Multi-Domain command and control conference", available at: www. afspc.af.mil/About-Us/Leadership-Speeches/Speeches/Display/Article/1311033/multi-domaincommand-and-control-conference/ (accessed 1 November 2020).

Rhodes, D. and Ross, A. (2010), "Five aspects of engineering complex systems", Paper presented at the IEEE Systems Conference, April, San Diego, CA, available at: https://ieeexplore.ieee.org/ document/5482431 (accessed 13 January 2021).

Richard, M., Ross, A., Stein, D. and Hastings, D. (2009), "Multi-Attribute tradespace exploration for survivability: Application to satellite radar", Paper presented at the AIAA SPACE 2009 Conference, September, Pasadena, CA, available at: http:/seari.mit.edu/documents/preprints/ RICHARDS_AIAA09.pdf (accessed 13 January 2021). 
JDAL

5,1

Schmunk, M.M. (2008), "Initial determination of low earth orbits using commercial telescopes", Master's Thesis, Air Force Institute of Technology.

Shell, J.R. (2010), "Optimizing orbital debris monitoring with optical telescopes", Paper presented at the Advanced Maui Optical Surveillance Conference, September, Kihei, HI, available at: https:// amostech.com/TechnicalPapers/2010/Systems/Shell.pdf (accessed 1 November 2020).

Trump, D.J. (2018), “Space policy directive 3, national space traffic management policy”, June.

USSTRATCOM (2004a), "Strategic command directive 505-1, space surveillance operations - basic operations, volume 1", p. 10.

USSTRATCOM (2004b), "Strategic command directive 505-1, space surveillance operations - basic operations, volume 2", p. 10.

Weeden, B. (2012), "Going blind: why America is on the verge of losing its situational awareness in space and what can be done about it", Secure World Foundation, pp. 14-15.

\section{Corresponding author}

Albert Vasso can be contacted at: albert.vasso@afit.edu

For instructions on how to order reprints of this article, please visit our website: www.emeraldgrouppublishing.com/licensing/reprints.htm

Or contact us for further details: permissions@emeraldinsight.com 